\title{
Efficient Statistical Simulation of Microwave Devices via Stochastic Testing-Based Circuit Equivalents of Nonlinear Components
}

\author{
Paolo Manfredi, Member, IEEE, Flavio G. Canavero, Fellow, IEEE
}

\begin{abstract}
This paper delivers a considerable improvement in the framework of the statistical simulation of highly nonlinear devices via polynomial chaos-based circuit equivalents. Specifically, a far more efficient and "black-box" approach is proposed that reduces the model complexity for nonlinear components. Based on recent literature, the "stochastic testing" method is used in place of a Galerkin approach to find the pertinent circuit equivalents. The technique is demonstrated via the statistical analysis of a low-noise power amplifier and its features in terms of accuracy and efficiency are highlighted.
\end{abstract}

Index Terms-Circuit simulation, microwave circuits, nonlinear circuits, polynomial chaos, power amplifiers, SPICE, statistical analysis, tolerance analysis, uncertainty.

\section{INTRODUCTION}

In recent years, strong attention has been drawn to the availability of techniques for the efficient inclusion of inherent parameter variability in the early-stage simulation of microwave and millimeter-wave electronic circuits [1]-[6]. As the technology is pushing towards further miniaturization, the impact of manufacturing process tolerances on highfrequency designs is becoming increasingly critical. However, the computational time for an accurate Monte Carlo (MC) analysis [7]-[9], available in virtually all circuit simulators, is often prohibitive due to the complex nature of the structures under investigation.

Although the methodologies in [1]-[6] aimed at lowering the computational burden associated to MC-like approaches, alternative modeling strategies that rely on the robust framework of polynomial chaos (PC) [10] were also proposed for microwave structures [11]-[15]. According to PC, stochastic responses are expanded in series of orthogonal polynomials that depend on the probability distribution of the random system parameters [16]. Although some trade-off exists, for a moderate number of random parameters, the determination of the PC expansion (PCE) coefficients is much faster than running a large number of $\mathrm{MC}$ simulations, and allows to efficiently obtain accurate statistical information on the system response.

This work was supported in part by the Research Foundation Flanders (FWO-Vlaanderen). P. Manfredi is currently an FWO Post-Doctoral Research Fellow.

P. Manfredi is with the Electromagnetics Group, Department of Information Technology, Ghent University, 9000 Gent, Belgium (e-mail: paolo.manfredi@ugent.be).

F. Canavero is with the Electromagnetic Compatibility Group, Department of Electronics and Telecommunications, Politecnico di Torino, 10129 Torino, Italy (e-mail: flavio.canavero@polito.it).
The calculation of the PCE coefficients is typically carried out via the solution of an augmented set of deterministic equations, built based on the random circuit properties via a stochastic Galerkin method (SGM) [17]. The authors of this contribution successfully applied the SGM to stochastic linear transmission-line problems [18]-[20]. As to circuitlevel simulations, this operation is relatively straightforward when dealing with linear circuits [21], whereas it required approximate relations to handle nonlinearities [22], since the presence of nonlinear functions impedes the use of orthogonality properties.

Some works recently extended the framework to general nonlinear circuits [23]-[25]. However, these approaches require the availability of a closed-form expression for the nonlinearity and their implementations are intrusive. In fact, a customized solver with ad-hoc device models needs to be developed. Such methods can hardly compete with commercial simulators, which offer hundreds of sophisticated device models whose internal description is often unavailable to the end user. Moreover, realistic designs sometimes include behavioral and/or encrypted models which cannot be handled by the approaches in [23]-[25].

To partially overcome this issue, a different modeling strategy was proposed in [26]. SPICE-compatible deterministic circuit models are created for each stochastic electrical element, connected in accordance with the original circuit topology, and simulated in a standard circuit simulator to obtain the soughtfor PCE coefficients. This non-intrusive approach allows to rely on the well-established solution algorithms and device models available in commercial software, and was applied e.g., to the simulation of power converters [27]. Nonetheless, as far as nonlinear elements are concerned, the implementation is based on the discretization of Galerkin projection integrals by means of Gaussian quadrature rules. Unfortunately, albeit rigorous and accurate, this technique does not scale favorably with the number of random variables (RVs) included, which limits the PC to the analysis of circuits with one or two random parameters only, while hindering the simulation of complex and realistic designs that include nonlinear components.

This paper improves the modeling methodology of nonlinear devices in [26]. The idea of stochastic testing (ST), introduced in [24], is accommodated in the state-of-the-art framework to mitigate the modeling complexity, thus leading to more efficient SPICE-compatible circuit equivalents that can be simulated without the need of a customized software. The preliminary assessment carried out in [28] is 
here complemented by means of comprehensive and in-depth mathematical derivations, comparisons against the state-ofthe-art tools, as well as discussions on the convergence and accuracy. Furthermore, it is shown how the generation of the models is independent of the physical description of the device, which makes the approach "black-box" and applicable to arbitrary multiport subcircuits. This circuit-based approach is fully compatible with any solver type, including transient and harmonic balance (HB). Finally, it is also detailed how worst-case (WC) responses can be conveniently estimated using the PC technique.

The paper is organized as follows: Section II summarizes the rationale of PC-based simulations and introduces the new proposed equivalent circuit models for nonlinear devices. In Section III, it is detailed how to estimate WC responses from the PCEs. The proposed modeling is validated in Section IV through the statistical simulation of a low-noise amplifier (LNA); a discussion on the efficiency is also provided. Finally, conclusions are drawn in Section V.

\section{Circuit Simulation via Polynomial Chaos}

This section briefly recalls the state-of-the-art approach for the non-intrusive PC simulation via circuit equivalents [26] and outlines the proposed improved modeling for nonlinear devices.

\section{A. The Polynomial Chaos Expansion}

The underlying idea of the PC-based simulation is to represent stochastic circuit voltages and currents as PCEs, e.g.,

$$
v(t)=\sum_{k=0}^{\infty} v_{k}(t) \varphi_{k}(\boldsymbol{\xi}) \approx \sum_{k=0}^{K-1} v_{k}(t) \varphi_{k}(\boldsymbol{\xi}),
$$

where $v$ denotes a generic voltage within the circuit and $v_{k}$ are the pertinent coefficients to be determined. According to the classical framework of PC [16], the series is truncated to a total number of

$$
K=\frac{(p+d) !}{p ! d !}
$$

terms. In the above equation, $d$ is the total number of RVs affecting the circuit, encompassed in the $d$-variate variable $\boldsymbol{\xi}$, whereas $p$ is the maximum polynomial degree, which can be tuned to achieve better accuracy. As shown e.g., in [26], and further demonstrated in this paper, choosing $p=2$ generally provides reasonable modeling accuracy.

The functions $\left\{\varphi_{k}\right\}$ in (1) are a $d$-variate polynomial basis which is orthonormal with respect to the inner product

$$
\langle f, g\rangle=\int_{\mathbb{R}^{d}} f(\boldsymbol{\xi}) g(\boldsymbol{\xi}) w(\boldsymbol{\xi}) d \boldsymbol{\xi},
$$

where $w(\boldsymbol{\xi})$ is the joint probability density function (PDF) of the RVs $\boldsymbol{\xi}$ [16]. For a detailed discussion on how to construct these multivariate bases, the reader is referred to [16] and [17].

Thanks to the orthogonality of the polynomials, the first PCE coefficient, e.g., $v_{0}(t)$, is the average response, whilst the sum of the squares of the remaining coefficients, e.g., $\sum_{k=1}^{K-1} v_{k}^{2}(t)$, provides an estimation of its variance. Other statistical information, like higher-order moments, distribution functions or quantiles, are readily extracted by randomly sampling (1) instead of running repeated and time-consuming circuit simulations.

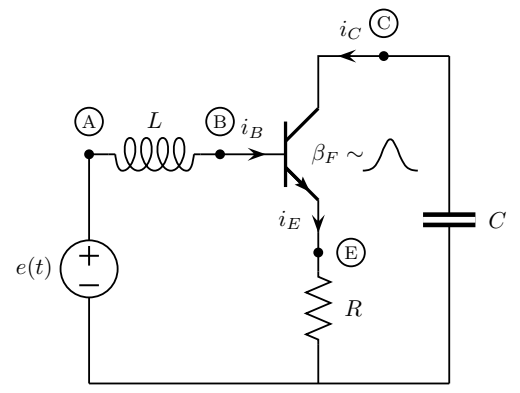

Fig. 1. Illustrative amplifier circuit including a BJT with random forward current gain $\beta_{F}$.

\section{B. The Deterministic Equivalent Augmented Network}

This section outlines the simulation procedure to calculate the PCE coefficients for circuit voltages and currents. The technique is illustrated based on the amplifier circuit in Fig. 1, which includes a bipolar junction transistor (BJT) and where the bias network is deliberately omitted to limit the network size. It is also assumed that the circuit is affected by one random parameter only, i.e., the forward current gain $\beta_{F}$ of the BJT, which has a Gaussian distribution with a nominal value of 145 and a relative standard deviation of $10 \%$. In this paper, which focuses on the more critical modeling of nonlinear devices, linear elements are considered as deterministic.

According to the PC framework, and for the sake of convenience and generality, the parameter $\beta_{F}$ is expressed in terms of a standardized RV:

$$
\beta_{F}=145+14.5 \xi,
$$

where $\xi$ is a Gaussian variable with zero mean and unit variance. Following the simulation strategy in [26], each stochastic node voltage and branch current is represented as a PCE like (1). Assuming for simplicity a two-term expansion $(K=2)$ leads e.g., to

$$
v_{B}(t) \approx v_{B 0}(t) \varphi_{0}(\xi)+v_{B 1}(t) \varphi_{1}(\xi)
$$

and

$$
i_{B}(t) \approx i_{B 0}(t) \varphi_{0}(\xi)+i_{B 1}(t) \varphi_{1}(\xi),
$$

where $\varphi_{0}=1$ and $\varphi_{1}=\xi$ are the first two Hermite polynomials [16]. Analogous representations hold for the other node voltages and branch currents.

A new node is then associated to each voltage coefficient, and these nodes are connected with suitable multi-terminal equivalent circuit models for each electrical element, as illustrated in Fig. 2 by means of the dashed shapes. These equivalent models must enforce a proper relationship between the terminal voltages and currents, so that the (deterministic) node voltages and branch currents in the equivalent network coincide with the PCE coefficients of the (stochastic) voltages and currents in the original network. However, the linear 
elements being in this case deterministic, their equivalent models reduce to a mere replication of the element itself. (The proper models to be used in the dashed boxes when the linear elements are also stochastic are discussed in [26] and are not repeated in the present paper.)

With the proper deterministic equivalents, the augmented circuit in Fig. 2 is simulated once to retrieve the PCE coefficients that provide pertinent statistical information without the need to perform many repeated $\mathrm{MC}$ simulations of the original circuit. A novel and more efficient approach to suitably model a nonlinear element like the BJT in Fig. 1 is discussed in the next section.

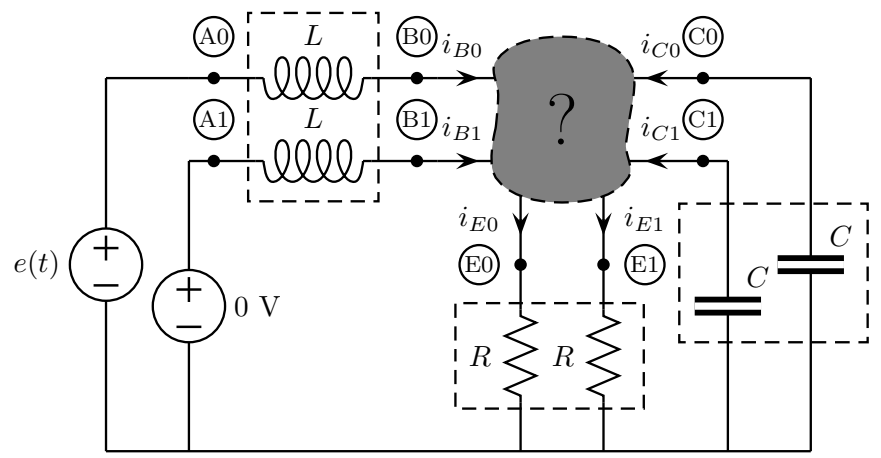

Fig. 2. Deterministic equivalent augmented counterpart of the circuit in Fig. 1. The node voltages and the branch currents correspond to the PCE coefficients of the stochastic voltages and currents in the original circuit.

\section{Stochastic Testing-Based Models for Nonlinear Devices}

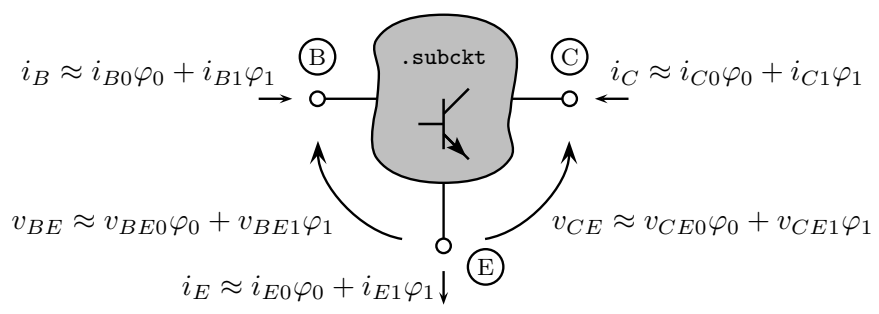

Fig. 3. Generic stochastic subcircuit describing a nonlinear device like the BJT in Fig. 1, with the pertinent terminal voltages and currents.

Consider Fig. 3, where the BJT symbol is replaced by the gray shape to emphasize that the transistor may be described by a generic subcircuit whose inner description is not necessarily available. The terminal voltages and currents are represented by their PCEs, where the dependence of the basis functions on $\xi$ has been dropped for notational convenience.

In order to find a suitable deterministic equivalent for the nonlinear transistor, the following two-step procedure is adopted:

Step \#1: Take $K=2$ instances of the stochastic element with distinct samples of its random parameter $\beta_{K}$, corresponding e.g., to the standardized samples $\xi_{0}=-1\left(\beta_{F}=130.5\right)$ and $\xi_{1}=+1\left(\beta_{F}=159.5\right)$, as shown in Fig. 4. For consistency, the terminal voltages and currents are also sampled in the corresponding value of $\xi$, and the notation

$$
a_{m k}=\varphi_{k}\left(\xi_{m}\right)
$$

(with $m, k=0,1$ ) has been introduced, leading to $a_{00}=$ $a_{10}=a_{11}=1$ and $a_{01}=-1$. It is important to point out that the two instances in Fig. 4 are deterministic, since the random parameter has been sampled at a specific value, and so are the terminal voltages and currents.

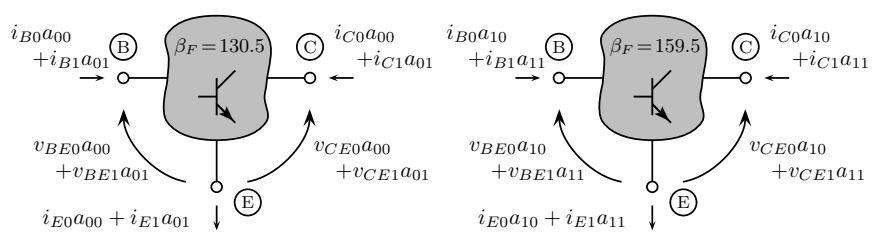

Fig. 4. Deterministic instances of the nonlinear device with sampled random parameters.

Step \#2: Express the currents at terminals B and C as

$$
\begin{aligned}
& j_{X 0}=i_{X 0} a_{00}+i_{X 1} a_{01} \\
& j_{X 1}=i_{X 0} a_{10}+i_{X 1} a_{11}
\end{aligned}
$$

$(X=B, C)$, and retrieve the current PCE coefficients as

$$
\begin{aligned}
& i_{X 0}=j_{X 0} b_{00}+j_{X 1} b_{01} \\
& i_{X 1}=j_{X 0} b_{10}+j_{X 1} b_{11},
\end{aligned}
$$

where

$$
\left[\begin{array}{ll}
b_{00} & b_{01} \\
b_{10} & b_{11}
\end{array}\right]=\left[\begin{array}{ll}
a_{00} & a_{01} \\
a_{10} & a_{11}
\end{array}\right]^{-1}=\left[\begin{array}{rr}
0.5 & 0.5 \\
-0.5 & 0.5
\end{array}\right] .
$$

It should be noted that, according to the Kirchhoff current law (KCL), the PCE coefficients of the current at the third terminal are given by

$$
\begin{aligned}
& i_{E 0}=i_{B 0}+i_{C 0} \\
& i_{E 1}=i_{B 1}+i_{C 1} .
\end{aligned}
$$

Equations (8) and (10) provide a relation between the current and voltage PCE coefficients through the instances in Fig. 4. The corresponding circuit equivalent implementing such equations is shown in Fig. 5 and is used in the circuit of Fig. 2 as the deterministic augmented model for the transistor.

It is important to remark that the equivalent model of Fig. 5 is implemented by means of standard circuit elements only, i.e., dependent sources and instances of the original stochastic element. Moreover, no particular assumption has been made on the internal description of the nonlinear device, which is indeed treated as a "black-box" component. By way of example, the HSPICE netlist for the augmented circuit of Fig. 2 with the equivalent model in Fig. 5 is provided in the Appendix A.

The outlined procedure is readily generalized to an arbitrary number of terms in the PCEs, by considering $K$ deterministic instances of the stochastic element, and to arbitrary multiterminal devices. In general, a clever and effective set of $K$ sampling points for the random element parameters is 
generated by means of the ST algorithm proposed in [24] and summarized in Appendix B. The sampling points are always given in terms of standardized $\mathrm{RVs}$, like $\xi$ in the discussed tutorial example. A $K \times K$ matrix with entries $a_{m k}$ as in (6) is constructed that collects the weights for the dependent voltages sources, and then inverted to obtain the corresponding weights $b_{m k}$ for the dependent current sources. The example section shows how this new ST-based implementation turns out to be accurate, yet way more efficient, than the previous SGM-based one.
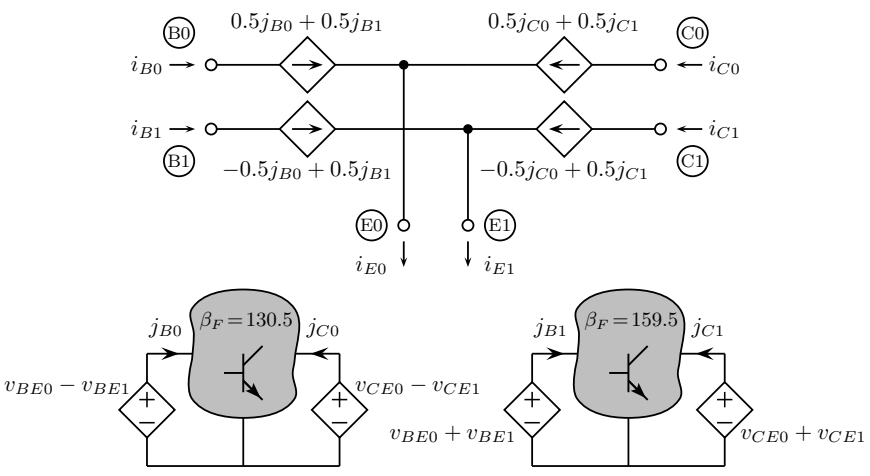

Fig. 5. Complete deterministic augmented equivalent of the BJT for a PCE with $K=2$ terms.

\section{Comparison with the Galerkin-Based Modeling}

The proposed modeling approach requires $K$ instances of the original stochastic element, where $K$ coincides with the number of unknowns (2) in each PCE. The state-of-the-art modeling of nonlinear devices [26] is instead based on a SGM and on the discretization of projection integrals by means of a tensor product Gaussian quadrature rule. This solution is accurate and rigorous, but also inefficient. In fact, contrary to the models for linear elements, it requires the inclusion of $Q=(p+1)^{d} \gg K$ instances of the original nonlinear element, thus rendering the model complexity hardly tractable when $d>2$. Although better quadrature schemes could in principle be adopted, their implementation is cumbersome. Hence, this methodology can hardly deal with multiple RVs.

Fig. 6 compares the model complexity of nonlinear devices for the novel and state-of-the-art approaches when $p=2$ and the number of RVs $d$ is increased. For $d=1, Q=K=3$; however, when $d>1$, the complexity of the previous implementation scales exponentially and is therefore outperformed by the new one. It should be noted that the model complexity for linear elements is already $K$ in [26]. Hence, although the novel method is in principle applicable to linear elements as well, it does not provide any relevant advantage.

\section{WORST-CASE RESPONSE}

Sometimes the designer is interested in estimating WC responses, i.e., in computing the upper and lower bounds of the output response. A brute-force but inefficient approach for WC analysis is to use a large number of MC samples, considering a bounded uniform distribution for the random parameters.

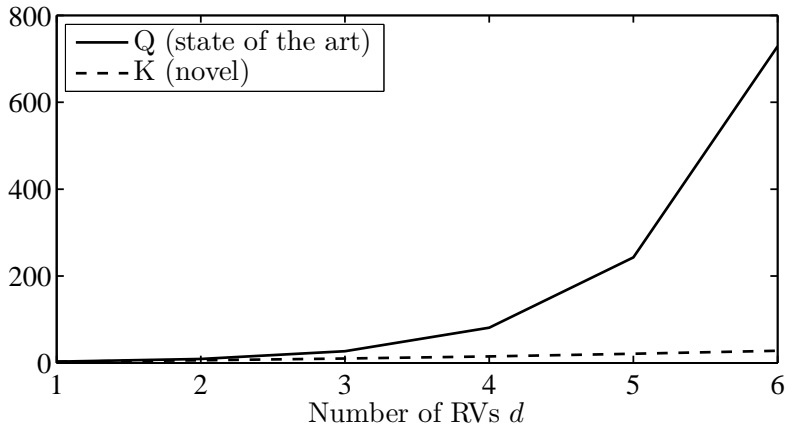

Fig. 6. Model complexity of nonlinear components for the state-of-theart [26] (solid line) and novel (dashed line) implementations, with $p=2$.

Smarter techniques make use e.g., of genetic algorithms [29]. However, it should be pointed out that a WC analysis is likely to provide unrealistic error margins, because the output responses are considered at their extremes, disregarding their low probability to occur.

Nevertheless, since the PCE (1) turns out to be in fact an analytical function of the RVs $\boldsymbol{\xi}$, it is possible to use it to estimate $\mathrm{WC}$ responses. The problem reduces to calculating the upper and lower bounds of the PCE and is carried out exactly when dealing with second-order expansions $(p=2)$, as shown in the following. The result is therefore exact within the approximation provided by the PCE. Although this implies an improper use of PC, because probability distributions are not taken into account, results consistent with $\mathrm{MC}$ simulations can be obtained, as shown in Section IV.

The calculation is carried out in a recursive manner. First, it is considered that a second-order polynomial may only have either one maximum or one minimum inside a bounded domain. The candidate point $\boldsymbol{\xi}_{\text {zero }}$ for the maximum or minimum is the point that nullifies the components of the gradient, i.e.,

$$
\nabla v=0
$$

where $v$ is intended as the PCE in (1). It should be noted that the derivatives in (11) always produce a first-order $d$-dimensional polynomial (i.e., a linear equation) in the multivariate variable $\boldsymbol{\xi}$. Hence, the solution of (11) is written in matrix form as

$$
\boldsymbol{\xi}_{\text {zero }}=\mathbf{D}^{-1} \mathbf{u},
$$

where $\mathbf{D}$ and $\mathbf{u}$ collect the pertinent coefficients arising from the gradient derivatives.

Besides this possible maximum or minimum, the PCE also has a minimum and/or maximum on the boundary. Hence, the $d$-dimensional boundaries are iteratively explored by evaluating the PCE at the extremes of $\boldsymbol{\xi}$. Each evaluation produces a second-order $(d-1)$-dimensional polynomial. Therefore, the new candidate points are again calculated via (11) and (12). The outlined procedure is applied recursively until the trivial case $d=1$ (parabola) is reached. At every iteration, it is checked whether the PCE values at the new candidate points are greater or smaller than the previously-computed solutions. 


\section{ApPlications AND Numerical Results}

This section validates the proposed modeling methodology through the simulation of the 2-GHz LNA in Fig. 7 [30], which uses a NXP BFG425W wideband BJT. The amplifier is supplied with a voltage of $4.5 \mathrm{~V}$. Tab. I collects all the data concerning the circuit components. The transmission lines are microstrip traces with a thickness of $2 \mu \mathrm{m}$ on a substrate with a relative permittivity of 4.6 and a thickness of $0.5 \mathrm{~mm}$. The BJT is modeled using a SPICE library available by the vendor, which in turn relies on a level-1 (Gummel-Poon) buildin HSPICE model. All the simulations are carried out using HSPICE on an ASUS U30S laptop with an Intel(R) Core(TM) i3-2330M, CPU running at $2.20 \mathrm{GHz}$ and $4 \mathrm{~GB}$ of RAM.

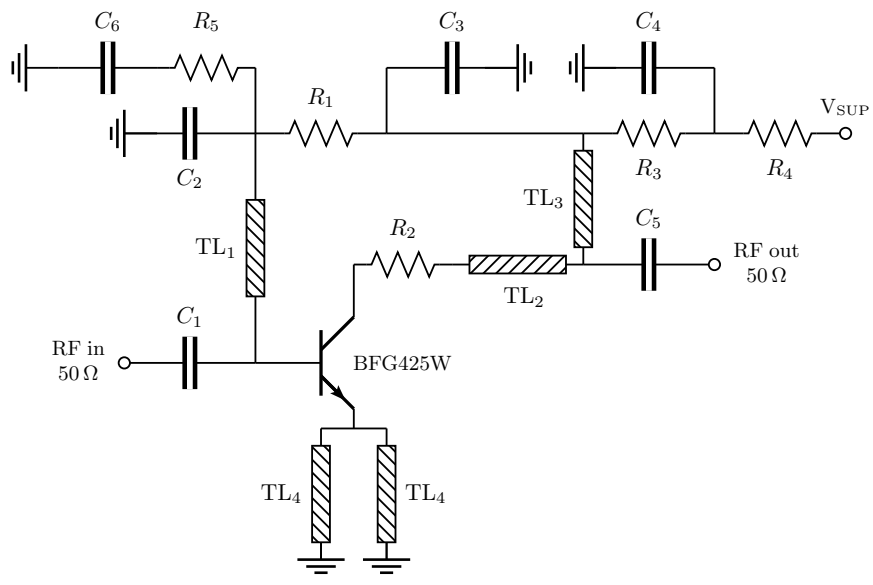

Fig. 7. 2-GHz BJT low-noise amplifier for the application example.

TABLE I

COMPONENT DATA FOR THE 2-GHz LNA.

\begin{tabular}{|c|c|c|c|}
\hline component & value & component & value \\
\hline \hline$R_{1}$ & $15 \mathrm{k} \Omega$ & $R_{2}$ & $0 \Omega$ \\
\hline$R_{3}$ & $22 \Omega$ & $R_{4}$ & $82 \Omega$ \\
\hline$R_{5}$ & $100 \Omega$ & & \\
\hline$C_{1}$ & $4.7 \mathrm{pF}$ & $C_{2}$ & $5.6 \mathrm{pF}$ \\
\hline$C_{3}$ & $5.6 \mathrm{pF}$ & $C_{4}$ & $1 \mathrm{nF}$ \\
\hline$C_{5}$ & $2.7 \mathrm{pF}$ & $C_{6}$ & $100 \mathrm{nF}$ \\
\hline $\mathrm{TL}_{1}$ & \multicolumn{3}{|l|}{ width: $0.25 \mathrm{~mm}$, length: $8.9 \mathrm{~mm}$} \\
\hline $\mathrm{TL}_{2}$ & \multicolumn{3}{|l|}{ width: $0.25 \mathrm{~mm}$, length: $3.9 \mathrm{~mm}$} \\
\hline $\mathrm{TL}_{3}$ & \multicolumn{3}{|l|}{ width: $0.25 \mathrm{~mm}$, length: $6.6 \mathrm{~mm}$} \\
\hline $\mathrm{TL}_{4}$ & \multicolumn{4}{|l}{ width: $0.80 \mathrm{~mm}$, length: $3.0 \mathrm{~mm}$} \\
\hline
\end{tabular}

\section{A. Transient Analysis}

A large-signal transient analysis is performed first. For this simulation, up to $d=2 \mathrm{RVs}$ are considered, namely the forward current gain and the substrate capacitance of the BJT. These parameters are assumed to be independent and Gaussian distributed, with average values of $\beta_{F}=145$ and $C_{J S}=667.5 \mathrm{fF}$, respectively. With respect to the discussion in
Section II, a second standardized Gaussian RV $\eta$ is introduced, so that the stochastic gain and capacitance are in general expressed as $\beta_{F}=145(1+\sigma \xi)$ and $C_{J S}=667.5(1+\sigma \eta) \mathrm{fF}$, where $\sigma$ denotes the relative standard deviation. The input signal is a sinusoid with a $+10-\mathrm{dBm}$ power and a frequency of $2 \mathrm{GHz}$.

To calculate statistical information via the proposed PCbased methodology, a single simulation of a $K$-augmented deterministic network is carried out. This equivalent circuit is automatically generated in MATLAB following the procedure outlined in Section II and analyzed in HSPICE. Being the linear elements non-stochastic, they are merely replicated $K$ times in the augmented circuit. The stochastic BJT is modeled using the proposed ST-based approach instead. It is worth mentioning that the available library model for the BFG425W is used transparently in the proposed simulation approach.

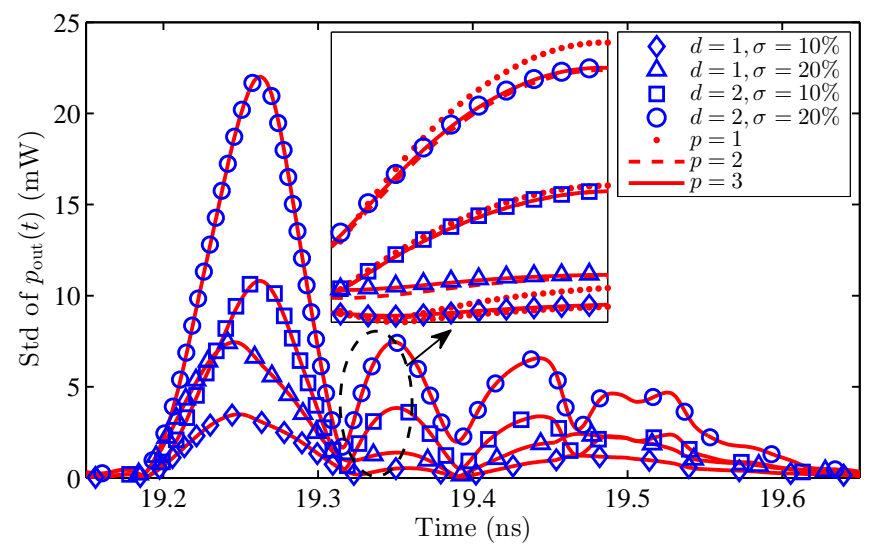

Fig. 8. Standard deviation of the steady-state time-domain output power for a sinusoidal input power of $+10 \mathrm{dBm}$. Markers: reference results from MC analysis by considering one RV with a relative standard deviation of $10 \%$ $(\diamond)$ or $20 \%(\triangle)$, or two RVs with the same independent variations ( $\square$ and $\bigcirc$, respectively); lines: results from the PC-based simulation with order one (dotted), two (dashed) and three (solid line).

Fig. 8 shows one period of the standard deviation of the output power at the steady state. In particular, four scenarios are analyzed, by considering variations on one random parameter only, i.e., $\beta_{F}$, or both $\beta_{F}$ and $C_{J S}$, as well as relative standard deviations $\sigma$ of either $10 \%$ or $20 \%$ for each parameter. The markers indicate the results obtained with a standard MC analysis with 10000 samples, whereas the solid line is the result from the PC-based simulation with order $p=3$. The PCE coefficients for the power are retrieved from the voltage and current coefficients as described in [28]. As shown by the figure, the magnitude of the standard deviation is of course larger when the variations are greater $(\sigma=20 \%)$. Reasonably, it further increases when two RVs are considered instead of one.

To better appreciate the convergence and accuracy of the PC-based simulation, a zoom-in is displayed, where the MC result is further compared with PC simulations of orders $p=1$ (dotted line) and $p=2$ (dashed line), in addition to the third-order expansion. This comparison shows that the results obtained with second- and third-order expansions are hardly distinguishable, even when $\sigma=20 \%$, whereas the first- 
order expansion is not always accurate. To further assess the convergence, Tab. II reports the normalized root-mean-square error (NRMSE) and the normalized maximum error (NME) between the PC and $\mathrm{MC}$ results (the normalization factor is the maximum swing). It is shown that using a second-order expansion already suffices to obtain a NRMSE below $1 \%$ and a NME below $2 \%$, except for the case $d=1$ and $\sigma=20 \%$. This can be explained by the fact that with $d=1$, just $p+1$ terms are considered for the PCEs, and the standard deviation is estimated out of $p$ terms only.

TABLE II

NRMSE AND NME BETWEEN MC ANALYSIS AND PC RESULTS WITH DIFFERENT EXPANSION ORDERS.

\begin{tabular}{|c|c|c|c|c|c|c|c|}
\cline { 3 - 8 } \multicolumn{2}{c|}{} & \multicolumn{3}{c|}{ NRMSE } & \multicolumn{3}{c|}{ NME } \\
\hline \multirow{2}{*}{$d$} & $\sigma$ & $p=1$ & $p=2$ & $p=3$ & $p=1$ & $p=2$ & $p=3$ \\
\hline \hline \multirow{2}{*}{1} & $10 \%$ & $1.30 \%$ & $0.45 \%$ & $0.44 \%$ & $5.22 \%$ & $1.25 \%$ & $1.19 \%$ \\
\cline { 2 - 8 } & $20 \%$ & $4.37 \%$ & $1.32 \%$ & $0.58 \%$ & $13.93 \%$ & $3.73 \%$ & $1.77 \%$ \\
\hline \multirow{2}{*}{2} & $10 \%$ & $0.67 \%$ & $0.53 \%$ & $0.47 \%$ & $2.28 \%$ & $1.54 \%$ & $1.47 \%$ \\
\cline { 2 - 8 } & $20 \%$ & $1.52 \%$ & $0.62 \%$ & $0.31 \%$ & $6.81 \%$ & $1.78 \%$ & $0.82 \%$ \\
\hline
\end{tabular}

Tab. III summarizes, for all the models used to generate the results in Fig. 8, the polynomial basis functions $\varphi_{k}(\boldsymbol{\xi})$, the standardized sampling points $\boldsymbol{\xi}_{m}$ for the random component parameters, and the corresponding weights $a_{m k}$ for the dependent voltage sources $(m, k=0, \ldots, K-1)$. When $d=2$, the bivariate $\mathrm{RV}$ is defined as $\boldsymbol{\xi}=(\xi, \eta)$. For the sake of convenience, the weights are given as the entries of a $K \times K$ matrix $\mathbf{A}$. The weights $b_{m k}$ for the dependent current sources are obtained as the entries of $\mathbf{B}=\mathbf{A}^{-1}$.

At this point, it is relevant to remark that the sampling points and the weights solely depend on the expansion order $p$, the number of RVs $d$, as well as on their distribution type. They are therefore problem-independent. The sampling points and the matrices $\mathbf{A}$ and $\mathbf{B}$ for a wide variety of problems can be pre-computed and stored into look-up tables.

\section{B. DC Analysis}

Secondly, a WC analysis on the DC response of the BJT is carried out. For this purpose, only variations on $\beta_{F}$ are considered (uniform within $[120,170]$ ), as of course the capacitance does not affect the DC behavior. For the analysis, the collector-emitter voltage $v_{C E}$ is swept from $0 \mathrm{~V}$ to $3 \mathrm{~V}$, whilst three values are considered for the base current $i_{B}$, i.e., $2 \mathrm{~mA}, 5 \mathrm{~mA}$ and $10 \mathrm{~mA}$. Owing to the uniform variability, orthonormal Legendre polynomials [31] are used for the PCEs, the first three being $\varphi_{0}=1, \varphi_{1}=\sqrt{3} \xi, \varphi_{2}=\frac{3 \sqrt{5}}{2} \xi^{2}-\frac{\sqrt{5}}{2}$. The corresponding standardized sampling points generated by the ST algorithm for a second-order PCE are $\xi_{0}=0$, $\xi_{1}=-0.775$, and $\xi_{2}=+0.775$.

The light area in Fig. 9 is a superposition of MC samples of the collector currents $i_{C}$ calculated with the given bias configurations. The thick lines show the WC bounds estimated from the PCEs of the collector currents in each configuration and match very well with the spread predicted via the MC analysis.

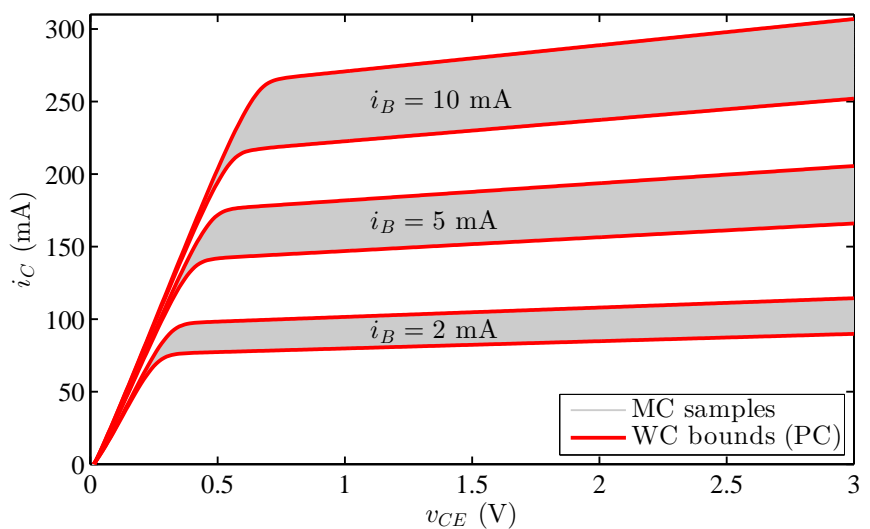

Fig. 9. BJT collector current in DC. Light area: superposition of MC samples providing the spread due to the variability of the forward current gain; thick lines: WC bounds estimated with PC.

\section{Harmonic Balance Analysis}

As a third test case, four RVs are considered. In addition to $\beta_{F}$ and $C_{J S}$, the base-emitter and base-collector junction capacitances are assumed to be also random with average values of $C_{J E}=310.9 \mathrm{fF}$ and $C_{J C}=137.7 \mathrm{fF}$, respectively. All the random parameters have a relative standard deviation of $10 \%$.

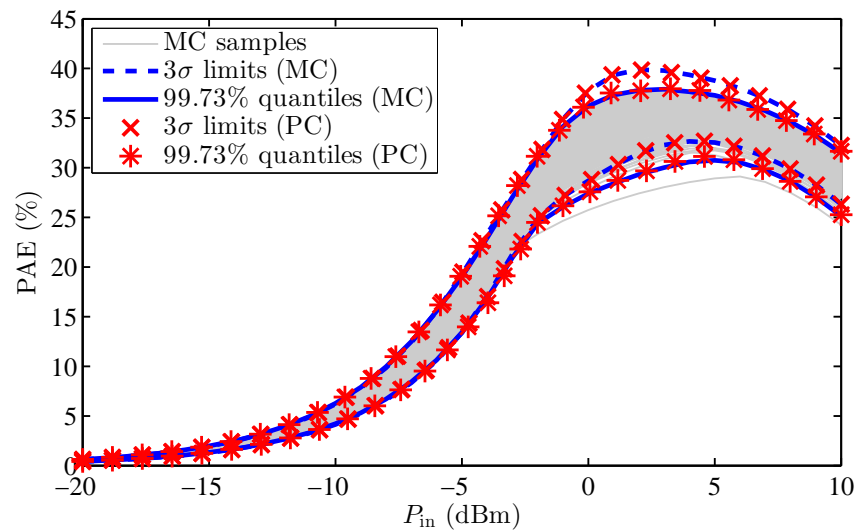

Fig. 10. Statistical assessment of the PAE for the LNA in Fig. 7. Thin lines: samples of the random response; thick lines: $3 \sigma$ limits (dashed) and $0.135 \%$ 99.865\% quantiles (solid) obtained with MC; markers: estimations provided by PC.

An HB analysis with 10 harmonics and a fundamental tone of $2 \mathrm{GHz}$ is performed with the HSPICE-RF tool. Fig. 10 shows the power added efficiency (PAE) as a function of the input power. The thin curves are a superposition of 1000 random responses from the MC analysis and provide a qualitative assessment of the response spread due to the variability. The dashed lines (the crosses) are the responses lying three times the standard deviation $\sigma$ from the average response, whilst the solid thick lines (the stars) are the responses corresponding to the $0.135 \%$ and $99.865 \%$ quantiles, all estimated with 10000 MC samples (with PC). A large number of samples is necessary to accurately capture the quantiles, thus rendering the MC simulation extremely inefficient. For the PC simulation, $p=2$ 
TABLE III

SAMPLING POINTS AND WEIGHTS OF THE DEPENDENT VOLTAGE SOURCES FOR THE MODELING OF THE BJT.

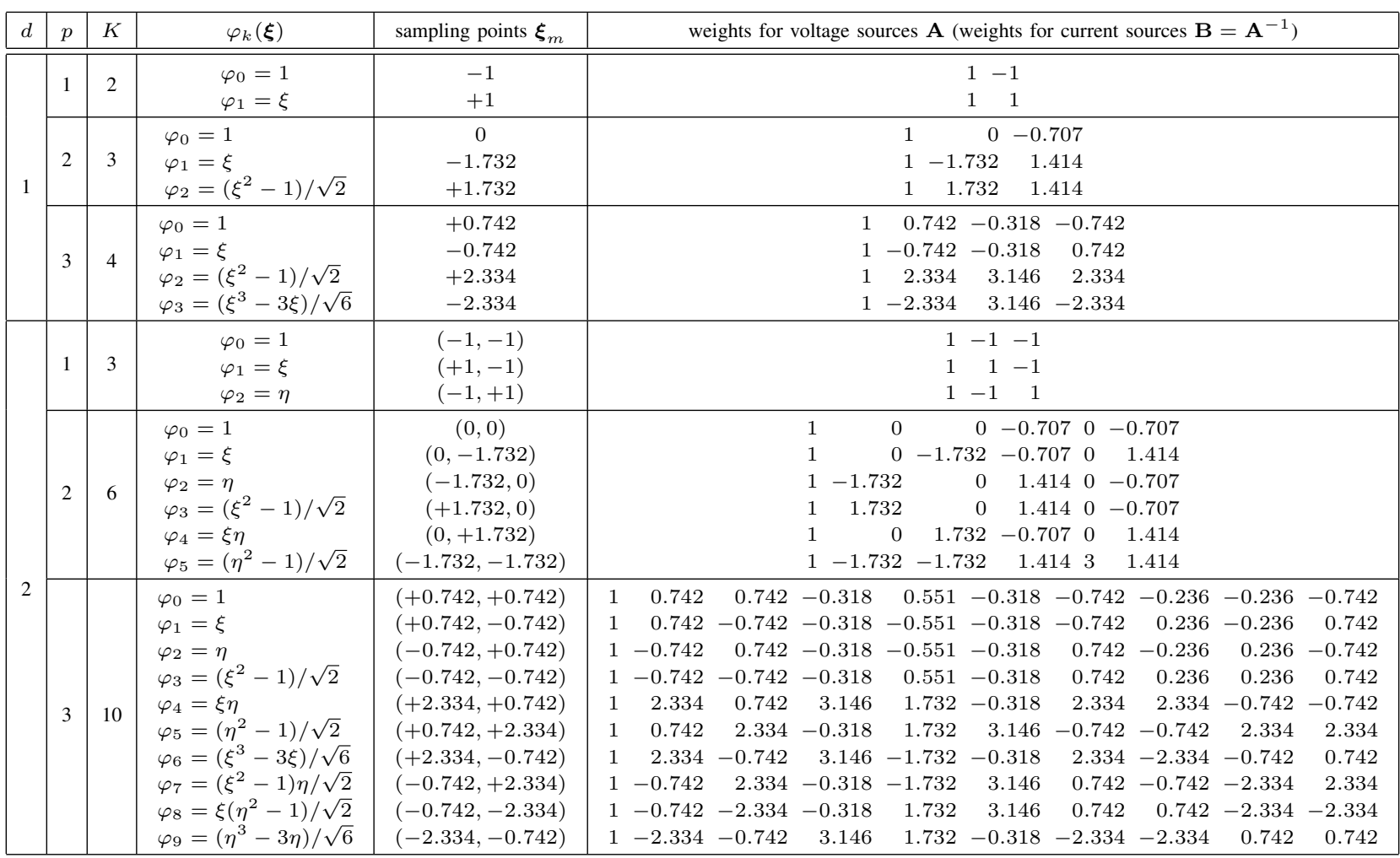

has been used. The two quantiles bound the $99.73 \%$ of the responses and coincide with $\pm 3 \sigma$ limits when the distribution on the response is Gaussian. The comparison between these curves indicates that for input powers above $-2 \mathrm{dBm}$, i.e., where the curves start to significantly differ, the PAE has no longer a Gaussian distribution. Therefore, average and standard deviation are not sufficient to fully characterize the strongly non-Gaussian response.

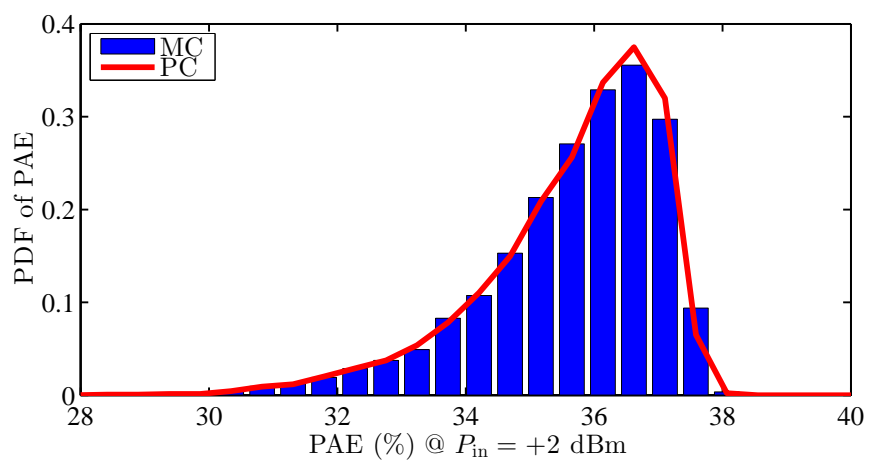

Fig. 11. PDF of the PAE for an input power of $+2 \mathrm{dBm}$. Bars: $\mathrm{MC}$ result; line: PC estimation.

For instance, Fig. 11 displays the PDF of the PAE for an input power of $+2 \mathrm{dBm}$, obtained with both $\mathrm{MC}$ (bars) and PC (line). From this plot it is possible to conclude that it is very unlikely that the amplifier exhibits an efficiency lower than $30 \%$ or larger than $38 \%$ for such an input power. From the above comparisons, the excellent accuracy provided by PC in reproducing the $\mathrm{MC}$ results is established.

In order to compare the accuracy achieved with the STbased implementation as opposed to the SGM-based modeling, Tab. IV collects the NMSE and NME between the two approaches and $\mathrm{MC}$ for both the $3 \sigma$ limits and the quantiles of Fig. 10. The results show that ST allows to obtain similar or even superior accuracy with respect to the SGM.

TABLE IV

ACCURACY ON THE PAE OBTAINED WITH BOTH THE SGM- AND ST-BASED MODELS.

\begin{tabular}{|c|c|c|c|c|}
\cline { 2 - 5 } \multicolumn{1}{c|}{} & \multicolumn{2}{c|}{ NRMSE } & \multicolumn{2}{c|}{ NME } \\
\hline quantity & SGM-based & ST-based & SGM-based & ST-based \\
\hline \hline $3 \sigma$ limits & $0.11 \%$ & $0.16 \%$ & $0.40 \%$ & $0.42 \%$ \\
\hline quantiles & $1.10 \%$ & $0.66 \%$ & $3.27 \%$ & $1.92 \%$ \\
\hline
\end{tabular}

As a further example, Fig. 12 reports a WC analysis of the time-domain output power for an input power of $+10 \mathrm{dBm}$. Once again, the thin curves are MC samples indicating the spread of the response due to the variations in the BJT parameters. The strongly non-sinusoidal behavior of the output power reveals that for such a large input the amplifier is highly nonlinear. The thick lines are the WC responses extracted from the PCE of the output power and match well the fluctuation bounds given by MC. For this WC simulation, the random parameters were ascribed a uniform distribution in the range 
$[-2 \sigma,+2 \sigma]$.

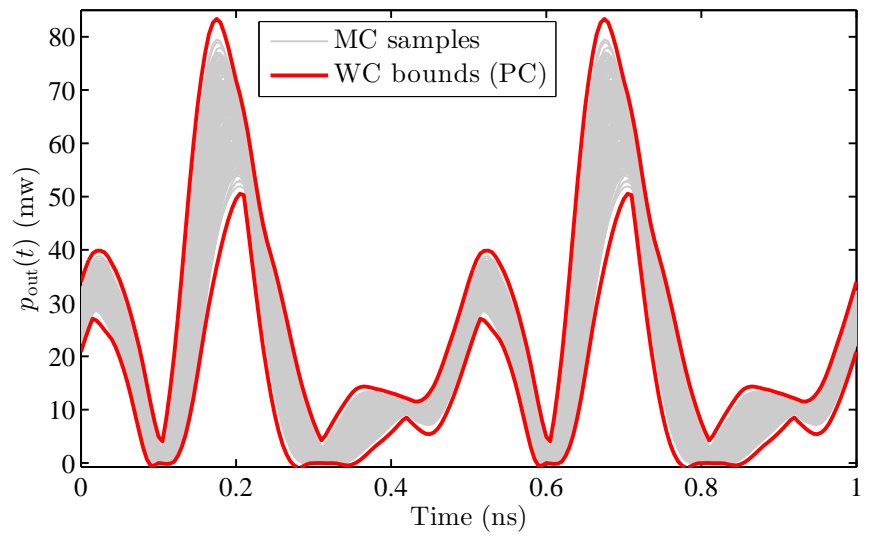

Fig. 12. Time-domain output power for an input power of $+10 \mathrm{dBm}$. Thin curves: samples of the random response; thick lines: WC bounds from the PCE of the output power.

\section{Small-Signal Frequency-Domain Analysis}

Finally, a small-signal frequency-domain (AC) analysis is carried out. In this simulation, three additional RVs are included, i.e., the base, collector and emitter inductances with averages $L_{C}=L_{B}=1.1 \mathrm{nH}$ and $L_{E}=0.25 \mathrm{nH}$, once again with a $10 \%$ Gaussian variation.

Fig. 13 displays the magnitude of $S_{11}$. The thin lines (from the MC analysis) show the very large fluctuation resulting from the variations of the seven random BJT parameters. In addition, the quantiles bounding the $95 \%$ and $99 \%$ of the responses, computed using both MC (dashed and solid thick lines, respectively) and PC with $p=2$ (crosses and stars), are shown. The accuracy of the PC result is again very good for this test case.

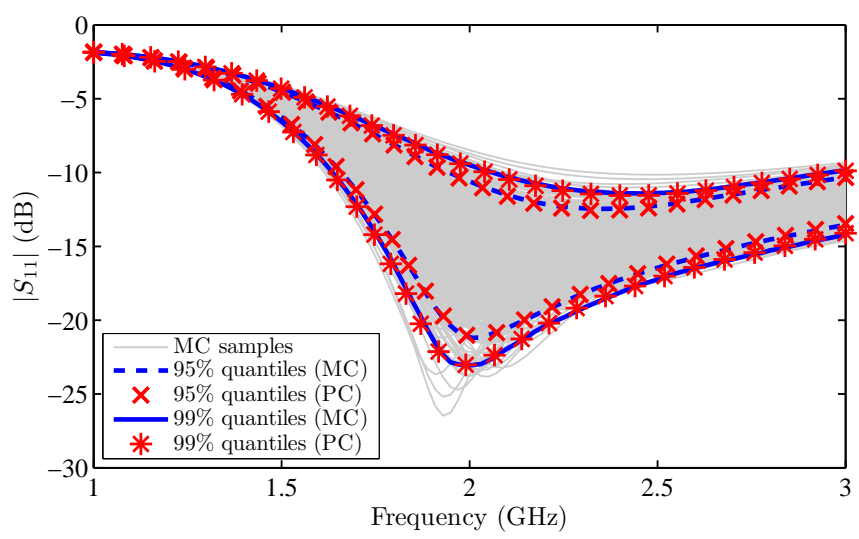

Fig. 13. Stochastic analysis of $\left|S_{11}\right|$. Thin lines: samples from MC analysis; dashed and solid thick lines: $95 \%$ and $99 \%$ quantiles, respectively, obtained from the MC samples; markers: same quantiles estimated via the PC-based circuit simulation.

\section{E. Efficiency Assessment}

Tab. V provides the main figures concerning the performance of the PC technique in conjunction with the new
ST-based circuit equivalents for the nonlinear elements. The simulation times are compared against a MC analysis with 10000 samples. Of course, the efficiency depends on the number of RVs included in the analysis and the order of the PCEs. However, for $p=2$, a speed-up of two orders of magnitude is achieved even when seven RVs are considered.

TABLE V

SIMULATION TIMES FOR THE CONSIDERED TEST CASES.

\begin{tabular}{|c|c|c|c|c|c|}
\hline analysis & $\mathrm{MC}$ (10k runs) & $d$ & $p$ & ST-based & speed-up \\
\hline \multirow{6}{*}{ TRAN } & \multirow{6}{*}{$13384.4 \mathrm{~s}$} & \multirow{3}{*}{1} & 1 & $2.8 \mathrm{~s}$ & $4700 \times$ \\
\hline & & & 2 & $3.8 \mathrm{~s}$ & $3500 \times$ \\
\hline & & & 3 & $5.2 \mathrm{~s}$ & $2500 \times$ \\
\hline & & \multirow{3}{*}{2} & 1 & $3.8 \mathrm{~s}$ & $3500 \times$ \\
\hline & & & 2 & $11.1 \mathrm{~s}$ & $1200 \times$ \\
\hline & & & 3 & $27.0 \mathrm{~s}$ & $490 \times$ \\
\hline HB & $11110.7 \mathrm{~s}$ & 4 & 2 & $64.9 \mathrm{~s}$ & $170 \times$ \\
\hline $\mathrm{AC}$ & $378.1 \mathrm{~s}$ & 7 & 2 & $3.7 \mathrm{~s}$ & $100 \times$ \\
\hline
\end{tabular}

To assess the efficiency improvement with respect to the state-of-the-art Galerkin-based models instead, Tab. VI collects the information about the complexity and simulation time of the two modeling approaches. The speed-up achieved with the novel implementation is consistent with the different complexity scale. The efficiency improvement becomes dramatic when $d$ is increased. With four RVs, the ST-based model is already more than one order of magnitude faster. No comparison is provided for the test case with seven RVs, as the complexity of the SGM-based model becomes intractable.

TABLE VI

EFFICIENCY COMPARISON BETWEEN SGM- AND ST-BASED MODELING.

\begin{tabular}{|c|c|c|r|r|r|r|}
\cline { 3 - 7 } \multicolumn{2}{c|}{} & \multicolumn{2}{c|}{ SGM-based } & \multicolumn{2}{c|}{ ST-based } & \multicolumn{1}{c|}{} \\
\hline$d$ & $p$ & complexity $Q$ & \multicolumn{1}{c|}{ time } & complexity $K$ & time & speed-up \\
\hline \hline \multirow{3}{*}{2} & 1 & 4 & $4.3 \mathrm{~s}$ & 3 & $3.8 \mathrm{~s}$ & $1.1 \times$ \\
\cline { 2 - 7 } & 2 & 9 & $13.1 \mathrm{~s}$ & 6 & $11.1 \mathrm{~s}$ & $1.2 \times$ \\
\cline { 2 - 7 } & 3 & 16 & $44.2 \mathrm{~s}$ & 10 & $27.0 \mathrm{~s}$ & $1.6 \times$ \\
\hline 4 & 2 & 81 & $870.2 \mathrm{~s}$ & 15 & $67.6 \mathrm{~s}$ & $12.9 \times$ \\
\hline 7 & 2 & 2187 & N/A & 36 & $3.7 \mathrm{~s}$ & - \\
\hline
\end{tabular}

\section{CONCLUSiOnS}

This paper improves the framework of the PC-based statistical simulation of highly nonlinear circuits, with specific emphasis on microwave devices. The simulation strategy relies on the analysis of an equivalent and deterministic, though augmented, counterpart of the original circuit with stochastic components. The equivalent models for nonlinear elements are constructed based on the ST technique in place of the previously used SGM. They consist of several deterministic instances of the stochastic component with sampled random parameters. The proposed modeling approach is far more efficient as the complexity scales better with the number 
of RVs. Furthermore, it is purely "black-box", thus being applicable to arbitrary multiport nonlinear subcircuits, even when the internal description is unavailable.

The simulation methodology is compatible with standard commercial SPICE-type simulators and any solver type. It allows to obtain accurate statistical information like moments, distribution functions and quantiles, with relevant speed-ups compared to both the classical MC method and the state-ofthe-art SGM-based modeling. In this paper, it is also detailed how WC responses can additionally be estimated with PC. Thorough validations in terms of accuracy and efficiency are provided via the statistical assessment of a low-noise power amplifier.

The present paper represents a step forward towards the simulation of complex nonlinear designs with a large number of random parameters. Inclusion of even larger numbers of RVs in PC simulations is, however, still an open issue and currently represents an active research field.

\section{ACKNOWLEDGMENT}

The authors would like to thank Dr. Riccardo Trinchero and Prof. Marco Pirola for the many fruitful discussions during the preparation of this work.

\section{APPENDIX A \\ SPICE NETLIST EXAMPLE}

The HSPICE netlist for the augmented circuit of Fig. 2 with the equivalent BJT model in Fig. 5 is provided below.

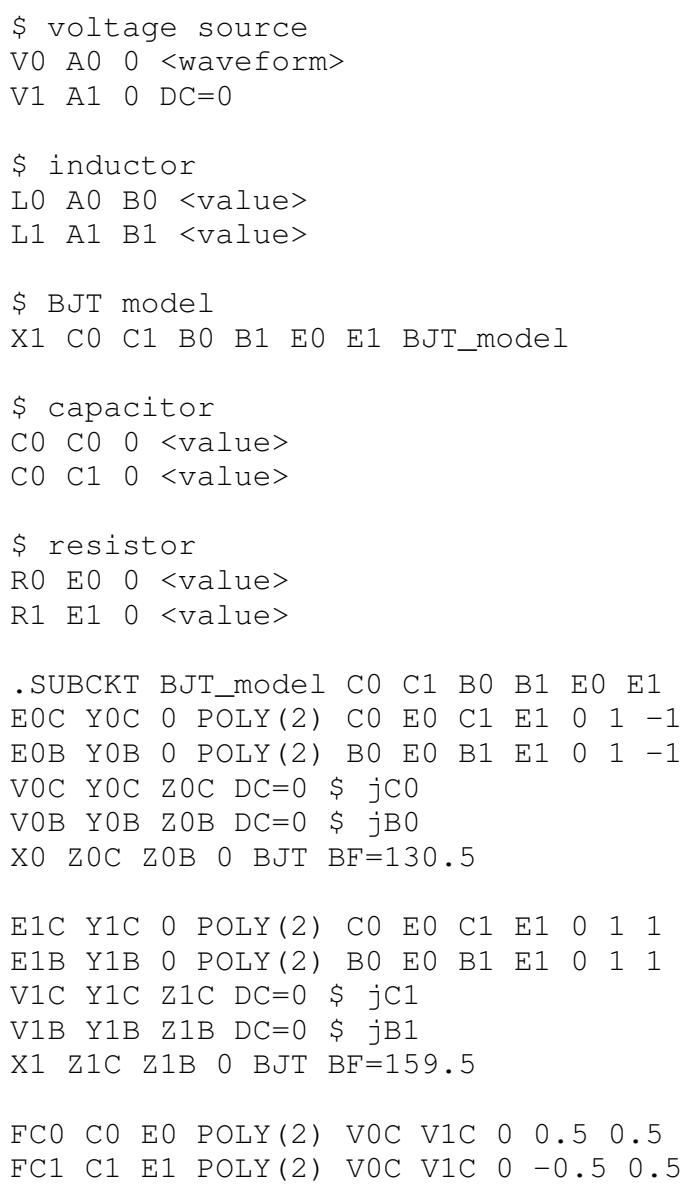

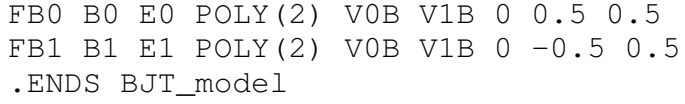

It is worth noting that dependent sources with multiple controlling variables are used for the weighted sums of voltages and currents.

\section{APPENDIX B}

\section{Generation of the SAMPling Points}

The set of $K$ sampling points $\left\{\boldsymbol{\xi}_{m}\right\}_{m=0}^{K-1}$ for the stochastic component parameters is selected based on the ST algorithm [24]. It is a subset of the $Q=(p+1)^{d}$ points $\left\{\boldsymbol{\zeta}_{q}\right\}_{q=0}^{Q-1}$ for a $d$-dimensional tensor product Gaussian quadrature rule [32]. The quadrature points are first sorted with decreasing order of the corresponding quadrature weights $\left\{w_{q}\right\}_{q=0}^{Q-1}$. The first quadrature point is then selected to be the first sampling point, i.e., $\boldsymbol{\xi}_{0}=\boldsymbol{\zeta}_{0}$. A corresponding $K \times 1$ matrix is constructed as

$$
\mathbf{M}=\frac{\tilde{\varphi}\left(\boldsymbol{\xi}_{0}\right)}{\left\|\tilde{\varphi}\left(\boldsymbol{\xi}_{0}\right)\right\|},
$$

where $\tilde{\boldsymbol{\varphi}}(\boldsymbol{\xi})=\left[\varphi_{0}(\boldsymbol{\xi}), \ldots, \varphi_{K-1}(\boldsymbol{\xi})\right]^{T}$.

Afterwards, an iterative procedure is carried out, in which a quadrature point $\boldsymbol{\zeta}_{q}$ is selected as an additional sampling point if the following inequality holds

$$
\frac{\left\|\mathbf{V}\left(\boldsymbol{\zeta}_{q}\right)\right\|}{\left\|\tilde{\boldsymbol{\varphi}}\left(\boldsymbol{\zeta}_{q}\right)\right\|}>\beta
$$

where

$$
\mathbf{V}\left(\boldsymbol{\zeta}_{q}\right)=\tilde{\varphi}\left(\boldsymbol{\zeta}_{q}\right)-\mathbf{M M}^{T} \tilde{\varphi}\left(\boldsymbol{\zeta}_{q}\right)
$$

whilst $\beta$ is a threshold coefficient (in this paper, $\beta=10^{-3}$ is used).

When a new sampling point is added, the $\mathbf{M}$ matrix is updated to include the normalized vector $\mathbf{V}\left(\boldsymbol{\zeta}_{q}\right)$

$$
\mathbf{M}=\left[\mathbf{M}, \mathbf{V}\left(\boldsymbol{\zeta}_{q}\right) /\left\|\mathbf{V}\left(\boldsymbol{\zeta}_{q}\right)\right\|\right]
$$

as a new column. The iteration is terminated when $K$ sampling points have been selected.

\section{REFERENCES}

[1] J. E. Rayas-Sánchez and V. Gutiérrez-Ayala, "EM-based Monte Carlo analysis and yield prediction of microwave circuits using linear-input neural-output space mapping," IEEE Trans. Microw. Theory Techn., vol. 54 , no. 12 , pp. $4528-4537$, Dec. 2006.

[2] L. Zhang, Q.-J. Zhang, and J. Wood, "Statistical neuro-space mapping technique for large-signal modeling of nonlinear devices," IEEE Trans. Microw. Theory Techn., vol. 56, no. 11, pp. 2453-2467, Nov. 2008.

[3] A. V. Sathanur, R. Chakraborty, and V. Jandhyala, "A hierarchical electromagnetic-circuit technique for statistical analysis of RF circuits in the spectral domain," IEEE Trans. Microw. Theory Techn., vol. 57, no. 4, pp. 796-806, Apr. 2009.

[4] L. Zhang, P. H. Aaen, and J. Wood, "Portable space mapping for efficient statistical modeling of passive components," IEEE Trans. Microw. Theory Techn., vol. 60, no. 3, pp. 441-450, Mar. 2012.

[5] J. Yao, Z. Ye, and Y. Wang, "Statistical analysis of process variations in RF/mm-wave circuits with on-the-fly passive macro-modeling," IEEE Trans. Microw. Theory Techn., vol. 61, no. 2, pp. 727-735, Feb. 2013.

[6] J. S. Ochoa and A. C. Cangellaris, "Random-space dimensionality reduction for expedient yield estimation of passive microwave circuits," IEEE Trans. Microw. Theory Techn., vol. 61, no. 12, pp. 4313-4321, Dec. 2013.

[7] R. Spence and R. S. Soin, Tolerance Design of Electronic Circuits. London: Imperial College Press, 1997. 
[8] J. F. Swidzinski and K. Chang, "Nonlinear statistical modeling and yield estimation technique for use in Monte Carlo simulations [microwave devices and ICs]," IEEE Trans. Microw. Theory Techn., vol. 48, no. 12, pp. 2316-2324, Dec. 2000

[9] A. Bhargava, "Statistical analysis of microwave circuits predicts real world performance," High Frequency Electron. Mag., pp. 16-23, Jan. 2007

[10] D. Xiu, "Fast numerical methods for stochastic computations: a review," Commun. Computational Physics, vol. 5, no. 2-4, pp. 242-272, Feb. 2009.

[11] P. Manfredi and F. G. Canavero, "Polynomial chaos-based tolerance analysis of microwave planar guiding structures", in IEEE MTT-S Int. Microwave Symp. Dig., Baltimore, MD, USA, Jun. 2011, pp. 1-3.

[12] A. Rong and A. C. Cangellaris, "Low-order macromodels of planar waveguides exhibiting geometric, material, and layout uncertainty", in IEEE MTT-S Int. Microwave Symp. Dig., Montreal, QC, Canada, Jun. 2012, pp. 1-3.

[13] D. Spina, F. Ferranti, T. Dhaene, L. Knockaert, G. Antonini, and D. Vande Ginste, "Variability analysis of multiport systems via polynomial-chaos expansion," IEEE Trans. Microw. Theory Techn., vol. 60, no. 8, pp. 2329-2338, Aug. 2012.

[14] A. C. M. Austin and C. D. Sarris, "Efficient analysis of geometrical uncertainty in the FDTD method using polynomial chaos with application to microwave circuits," IEEE Trans. Microw. Theory Techn., vol. 61, no. 12 , pp. 4293-4301, Dec. 2013.

[15] D. Spina, D. De Jonghe, D. Deschrijver, G. Gielen, L. Knockaert, and T. Dhaene, "Stochastic macromodeling of nonlinear systems via polynomial chaos expansion and transfer function trajectories," IEEE Trans. Microw. Theory Techn., vol. 62, no. 7, pp. 1454-1460, Jul. 2014.

[16] D. Xiu and G. E. Karniadakis, "The Wiener-Askey polynomial chaos for stochastic differential equations," SIAM J. Scientific Computing, vol. 24, no. 2, pp. 619-644, 2002.

[17] R. G. Ghanem and P. D. Spanos, Stochastic Finite Elements. A Spectral Approach. New York: Springer-Verlag, 1991.

[18] D. Vande Ginste, D. De Zutter, D. Deschrijver, T. Dhaene, P. Manfredi, and F. G. Canavero, "Stochastic modeling-based variability analysis of on-chip interconnects," IEEE Trans. Compon. Packag. Manuf. Techol., vol. 2, no. 7, pp. 1182-1192, Jul. 2012.

[19] P. Manfredi, D. Vande Ginste, D. De Zutter, and F. G. Canavero, "Improved polynomial chaos discretization schemes to integrate interconnects into design environments," IEEE Microw. Wireless Compon. Lett., vol. 23, no. 3, pp. 116-118, Mar. 2013.

[20] P. Manfredi, D. Vande Ginste, D. De Zutter, and F. G. Canavero, "Uncertainty assessment of lossy and dispersive lines in SPICE-type environments," IEEE Trans. Compon. Packag. Manuf. Techol., vol. 3, no. 7, pp. 1252-1258, Jul. 2013

[21] Q. Su and K. Strunz, "Stochastic circuit modelling with Hermite polynomial chaos," IET Electronics Letters, vol. 41, no. 21, pp. 1163 1165 , Oct. 2005.

[22] K. Strunz and Q. Su, "Stochastic formulation of SPICE-type electronic circuit simulation using polynomial chaos," ACM Trans. Model. Comput. Simul., vol. 18, no. 4, pp. 15:1-15:23, Sep. 2008.

[23] A. Biondi, D. Vande Ginste, D. De Zutter, P. Manfredi, and F. G. Canavero, "Variability analysis of interconnects terminated by general nonlinear loads," IEEE Trans. Compon. Packag. Manuf. Techol., vol. 3, no. 7, pp. 1244-1251, Jul. 2013.

[24] Z. Zhang, T. A. El-Moselhy, I. M. Elfadel, and L. Daniel, "Stochastic testing method for transistor-level uncertainty quantification based on generalized polynomial chaos," IEEE Trans. Comput.-Aided Des. Integr. Circuits Syst., vol. 32, no. 10, pp. 1533-1545, Oct. 2013.

[25] M. R. Rufuie, E. Gad, M. Nakhla, and R. Achar, "Generalized Hermite polynomial chaos for variability analysis of macromodels embedded in nonlinear circuits," IEEE Trans. Compon. Packag. Manuf. Techol., vol. 4, no. 4, pp. 673-684, Apr. 2014.

[26] P. Manfredi, D. Vande Ginste, D. De Zutter, and F. G. Canavero, "Stochastic modeling of nonlinear circuits via SPICE-compatible spectral equivalents," IEEE Trans. Circuits Syst. I, Reg. Papers, vol. 61, no. 7, pp. 2057-2065, Jul. 2014.

[27] P. Manfredi, I. S. Stievano, and F. G. Canavero, "Stochastic analysis of switching power converters via deterministic SPICE equivalents," IEEE Trans. Power Electron., vol. 29, no. 9, pp. 4475-4478, Sep. 2014.

[28] P. Manfredi and F. G. Canavero, "Statistical analysis of power microwave circuits via polynomial chaos", IEEE MTT-S Int. Microwave Symp. Dig., Tampa Bay, FL, USA, Jun. 2014, pp. 1-3.

[29] N. Fermia and G. Spagnuolo, "True worst-case circuit tolerance analysis using genetic algorithms and affine arithmetic," IEEE Trans. Circuits Syst. I, Fundam. Theory Appl., vol. 47, no. 9, pp. 1285-1296, Sep. 2000.
[30] T. Buss, "2GHz low noise amplifier with the BFG425W," Philips Semiconductors B.V., Nijmegen, The Netherlands, Application Note RNRT45-96-B-773, Nov. 1996.

[31] M. Abramowitz and I. A. Stegun, Handbook of Mathematical Functions (Applied Mathematics Series 55), 9th ed. New York: Dover, 1970.

[32] G. H. Golub and J. H. Welsch, "Calculation of Gauss quadrature rules," Mathematics of Computation, vol. 23, pp. 221-230, 1969.

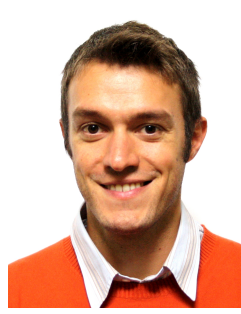

Paolo Manfredi (S'10-M'14) was born in 1985. He received the M.Sc. degree in Electronic Engineering from Politecnico di Torino, Turin, Italy, in 2009, and the Ph.D. degree in Information and Communication Technology from Scuola Interpolitecnica di Dottorato, within Politecnico di Torino, in 2013 $\mathrm{He}$ is currently a Postdoctoral Research Fellow of the Found for Scientific Research - Flanders (FWO) within the Electromagnetics Group at the Department of Information Technology (INTEC) of Ghent University, Ghent, Belgium. He was a Visiting Scientist with the Center for Advanced Life Cycle Engineering (CALCE), University of Maryland at College Park (UMCP), MD, USA, in June 2011 and from February to March 2012, and with the Department of Information Technology, Ghent University, from April to July 2012 and from May to June 2013. From March 2013 to September 2014 he was a Postdoctoral Researcher with the Electromagnetic Compatibility Group, Department of Electronics and Telecommunications, Politecnico di Torino. His research interests comprise circuit modeling, electromagnetic compatibility, nonuniform transmission lines, signal integrity, and statistical circuit simulation. Dr. Manfredi was selected for the IBM Best Student Recognition Event in 2009. He was the recipient of the Best Student Paper Award and Best Oral Paper Award at the 19th and 22nd IEEE Conference on Electrical Performance of Electronic Packaging and Systems (EPEPS) in 2010 and 2013, respectively, of an honorable mention from the IEEE MTT-S International Microwave Symposium (IMS) in 2011, and he was a Young Scientist Awardee at the XXX General Assembly and Scientific Symposium of the International Union of Radio Science (URSI GASS) in 2011.

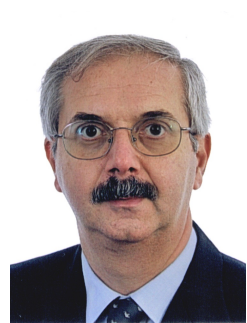

Flavio G. Canavero (SM'99-F'07) received his electronic engineering degree from Politecnico (Technical University) of Torino, Italy, and the Ph.D. degree from the Georgia Institute of Technology, Atlanta, USA, in 1986. Currently he is a Professor of Circuit Theory with the Department of Electronics and Telecommunications, Politecnico di Torino, where he serves also as the Director of the Doctoral School. He is an IEEE Fellow. He has been the Editor-in-Chief of IEEE Transactions on Electromagnetic Compatibility, V.P. for Communication Services of the EMC Society and Chair of URSI Commission E. He has been the Co-Chair, with Professor Clayton Paul, of the first edition of Global University in 2007. He received several Industry and IEEE Awards, including the prestigious Richard R. Stoddard Award for Outstanding Performance, which is the EMC Society's highest technical award. His research interests include signal integrity and EMC design issues, interconnect modeling, blackbox characterization of digital integrated circuits, EMI and statistics in EMC. 